\title{
INCLUSIVE JET PRODUCTION IN DEEP INELASTIC SCATTERING AT HIGH $Q^{2}$ AT HERA
}

\author{
STEPHEN MAXFIELD \\ Department of Physics, University of Liverpool, Liverpool, UK \\ H1 COLLABORATION
}

\begin{abstract}
A preliminary analysis of inclusive jet production in deep inelastic positron proton scattering at HERA is presented. The data sample corresponds to an integrated luminosity of $61.25 \mathrm{pb}^{-1}$ and was taken in the years 1999-2000 at a centre-of-mass energy $\sqrt{s}_{\mathrm{s}}=319 \mathrm{GeV}$ with the $\mathrm{H} 1$ detector. The measured jet cross section is compared to perturbative QCD calculations using the NLO program NLOJET++ and the strong coupling constant $\alpha_{\mathrm{s}}$ is extracted.
\end{abstract}

\section{Introduction}

Jet production at HERA in neutral current (NC) deep inelastic scattering (DIS) provides an important testing ground for QCD. Jet data are precise at high transverse energy, where experimental systematic uncertainties and nonperturbative effects are small. Here, we report on a measurement of the inclusive jet cross section in NC DIS at high momentum transfers $\mathrm{Q}^{2}$ and a determination of the strong coupling constant $\alpha_{S}\left(M_{Z}\right)$.

The kinematics of DIS are defined via the four momenta of the incoming $(k)$ and outgoing electron $\left(k^{\prime}\right)$, incoming proton $(p)$ and exchanged boson $q$ :

$$
\begin{array}{ll}
s=(k+p)^{2} \simeq 4 E_{e} E_{p} & Q^{2}=-q^{2}=-\left(k-k^{\prime}\right)^{2}, \\
x=Q^{2} / 2 p q, & y=Q^{2} / s x
\end{array}
$$

where $s$ is the centre-of-mass energy, $Q^{2}$ the negative four momentum transfer squared, $x$ the Bjørken scaling variable, and $y$ the inelasticity. In common with a previously published $\mathrm{H} 1$ analysis [1], this study uses the electron- $\Sigma$ method to calculate these kinematic variables.

To guarantee the factorisation property for the calculation of jet production in DIS, we analyse the events in the Breit frame of reference [2]. In this frame, selecting jets with high transverse energy $\left(E_{t}\right)$ suppresses contributions from the Born level (and jets induced by the beam remnant) ensuring the lowest order contributions to jet production are of $\mathcal{O}\left(\alpha_{S}\right)$. The sensitivity of the inclusive 
differential cross sections $d^{2} \sigma_{j e t} / d Q^{2} d E_{t}, d \sigma_{j e t} / d Q^{2}$ and $d \sigma_{j e t} / d E_{t}$ to $\alpha_{S}\left(M_{Z}\right)$ is exploited to make a determination of the constant.

\section{Event Selection, Jet Reconstruction and Correction Procedures}

The analysis uses data taken with the H1 detector [3] between 1999 and 2000, corresponding to an integrated luminosity of $61.25 \mathrm{pb}^{-1}$ at a centre-of-mass energy $\sqrt{s}=319 \mathrm{GeV}$. High $Q^{2}$ DIS events are identified by the detection of a scattered electron in the liquid argon calorimeter. Jets are found with the $\mathrm{k}_{\mathrm{t}}$ algorithm [4] in the Breit frame. The main selection cuts are listed below.

1) $150<Q^{2}<5000 \mathrm{GeV}^{2}$ and $0.2<y<0.6$.

2) Scattered electron energy $E_{e}{ }^{\prime}>11 \mathrm{GeV}$ and angle with respect to the proton beam, $\theta_{e}<153^{\circ}$.

3) $45<\sum\left(E-p_{z}\right)<65 \mathrm{GeV}$, where the sum is over all hadronic final state particles and the electron.

4) Jets with $E_{t}>7 \mathrm{GeV}$ and $-1.0<\eta_{\text {lab }}<2.5$ are selected.

Conditions 1) and 4) determine the phase space for the measured cross sections. The cross sections were extracted by correcting the data for limited detector acceptance, resolution and QED radiative effects. Bin-by-bin correction factors were determined from the Monte Carlo event generators DjangoH[5] 1.2 (using the Color Dipole Model with Ariadne[6]) and Rapgap[7] 2.8 (LO matrix elements plus parton showers) together with a detailed simulation of the H1 detector. Both, Rapgap and Django describe the shape of the distributions from the inclusive jet sample and the correction factors differ by less than $20 \%$ in most bins. Data were corrected using the mean of the two simulations and the model uncertainty taken to be half the difference. Uncertainties in the LAr calorimeter energy scale provide the other major source of systematic error.

\section{NLO QCD Calculations.}

In the following, the data are compared with NLO QCD calculations made by the program NLOJET++ [5] which have been corrected for hadronisation effects (typically $<10 \%$ ) using Django and Rapgap. The NLOJET++ calculations were made in the $\overline{M S}$ scheme, and $E_{t}{ }^{2}$ and $Q^{2}$ used for the renormalisation and factorisation scales respectively. CTEQ5M1 proton PDF's, $\alpha_{s}$ evolution to 2-loop precision and 5 active flavours were used. The theory uncertainties quoted contain contributions from the hadronisation corrections 
and from variation of the renormalisation and factorisation scales by the canonical factor of two.

\section{Results: Inclusive jet cross sections.}

Figure 1 shows the inclusive jet cross section as a function of the jet $E_{t}$ in 4 ranges of $\mathrm{Q}^{2}$. Note the accuracy of the NLO predictions over the whole measured phase space.

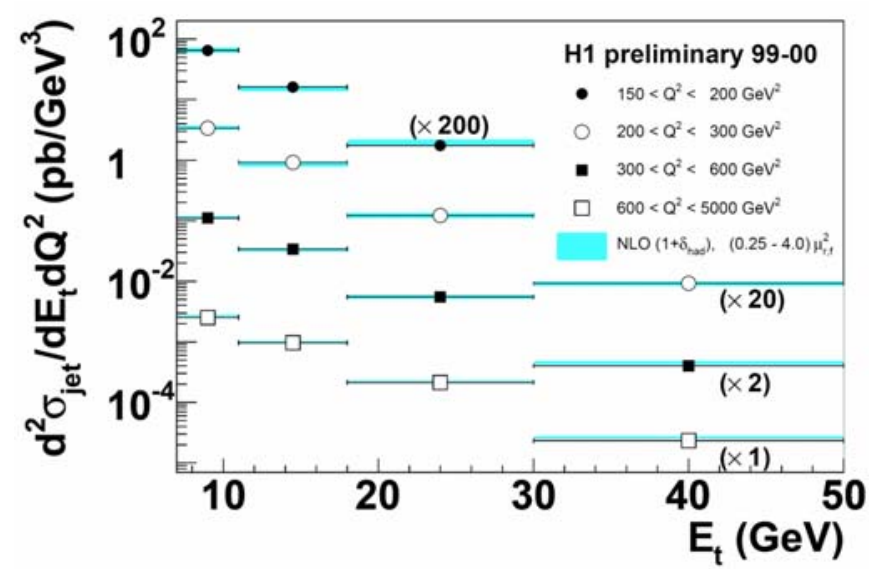

Figure 1. $d \sigma / d E_{t}$ in four bins of $Q^{2}$. The data are compared with NLOJET++ corrected for hadronisation effects. The shaded bands indicate the uncertainty arising from varying the renormalisation and factorisation scales by factors of 2 and from the hadronisation correction.

\section{Results: Determination of $\alpha_{\mathrm{S}}\left(M_{\mathrm{Z}}\right)$ and conclusions.}

Values of $\alpha_{\mathrm{S}}$ were extracted from the data using the methods given in [1,9]. The dependence of the cross section on $\alpha_{S}\left(M_{Z}\right)$ was determined by performing NLOJET++ calculations for a set of 5 values of $\alpha_{S}\left(M_{Z}\right)$ in each bin of $E_{t}$ and $Q^{2}$. The results of the calculations were then used to determine a set of functions, $\sigma_{i}\left(\alpha_{S}\left(M_{Z}\right)\right)=A_{i} \cdot \alpha_{S}\left(M_{Z}\right)+B_{i} \cdot \alpha^{2}{ }_{S}\left(M_{Z}\right)$, which provide a mapping between the measured cross section in each bin and a value of $\alpha_{S}\left(M_{Z}\right)$. Figure 2 shows the results of this procedure, in bins of $E_{t}$. The figure also shows (triangles) the results of a 2-loop evolution these $\alpha_{S}\left(M_{Z}\right)$ values to the mean $E_{t}$ of the data in each bin. The separate determinations of $\alpha_{S}\left(M_{Z}\right)$ are consistent over the whole $Q^{2}, E_{t}$ range and so all 15 measurements can be combined, taking due account of correlated uncertainties $[1,10]$, into a single measurement. The resulting average: 


$$
\left.\alpha_{S}\left(M_{Z}\right)=0.1197 \pm 0.0016 \text { (exp. }\right)_{-0.0048}^{+0.0046} \text { (th.) }
$$

is consistent with the world average and previous measurements from $\mathrm{H} 1$.

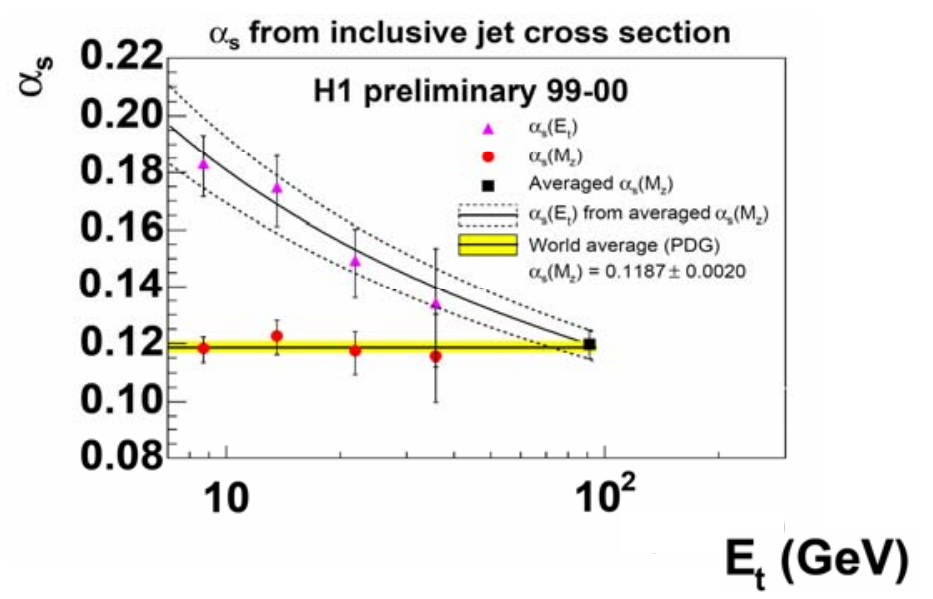

Figure 2. Measured values of $\alpha_{S}\left(M_{Z}\right)$ in bins of $E_{t}$ together with the result of evolving them with the 2-loop solution to the renormalisation group equation to obtain $\alpha_{S}\left(E_{t}\right)$. The horizontal line and shaded band represents the world average value of $\alpha_{S}\left(M_{Z}\right)$ and its error. The solid curve is the result of evolving from the value of $\alpha_{S}\left(M_{Z}\right)$ averaged over the 15 bins in $Q^{2}, E_{t}$ and the dashed curves indicate the extent of the corresponding errors.

\section{References}

1. H1 Collaboration, C. Adloff et al., Eur.Phys.J. C19 (2001), 1729: M. Wobisch, Ph.D thesis, PITHA 00/12.

2. B.R.Webber, J. Phys.. G19, (1993) 1567.

3. H1 Collaboration,I.Abt et al., Nucl. Instr. Meth. A386 (1997) 310 and 348.

4. S. Catani, Yu.L.Dokshitzer, M.H.Seymour and B.R.Webber, Nucl. Phys. B406 (1993) 187.

5. G. A. Schuler and H. Spiesberger, Proceedings of the Workshop Physics at HERA, vol. 3, eds. W. Buchm“uller, G. Ingelman, DESY (1992) 1419.

6. H. Jung, Comp. Phys. Comm. 86 (1995) 147.

7. L. Lönnblad, Comp. Phys. Comm. 71 (1992) 15.

8. Z. Nagy and Z. Trocsanyi, Phys. Rev. Lett. 87, (2001) 082001.

9. ZEUS Collaboration, J. Breitweg et al., Phys. Lett. B507 (2001) 70. E.Tassi Ph.D thesis DESY-THESIS-2001-059 (2001).

10. Lobo, C.Pascaud and F.Zomer, H1-01/98-536.

11. PDG Collaboration, S.Eidelman et al.,Phys. Lett B592 (2004) 1. 